\title{
Accuracy of HER2 status determination on breast core-needle biopsies (immunohistochemistry, FISH, CISH and SISH vs FISH)
}

Laurent Arnould ${ }^{1}$, Pascal Roger ${ }^{2}$, Gaëtan MacGrogan ${ }^{3}$, Marie-Pierre Chenard ${ }^{4}$, André Balaton $^{5}$, Sophie Beauclair ${ }^{6}$ and Frederique Penault-Llorca ${ }^{7}$, on behalf of CRITHER participant pathologists

${ }^{1}$ Biology and Tumor Pathology Department, Centre Georges-François Leclerc, Dijon, France; ${ }^{2}$ Anatomy and Cytology Department, Hôpital Lapeyronie, Montpellier, CHU Nîmes, Nîmes, France; ${ }^{3}$ Anatomy and Pathology Department, Institut Bergonié, Bordeaux, France; ${ }^{4}$ Pathology Department, Hôpital Hautepierre, Strasbourg, France; ${ }^{5}$ Cabinet d'Anatomopathologie, Bièves, France; ${ }^{6}$ Laboratoire Roche Pharma, Boulogne-Billancourt, France and ${ }^{7}$ Pathology Department, Centre Jean Perrin, Clermont-Ferrand, France

Preoperative breast cancer diagnosis on core biopsies has become a standard of care in many countries. Controversies exist concerning the accuracy of HER2 testing on biopsies as compared with surgical specimens, and few data exist concerning the use of emerging technologies such as bright-field in-situ hybridization in such a setting. A French multicenter, cross-sectional, histopathological study assessed the concordance of HER2 status determined by immunohistochemistry and silver (SISH) or chromogenic in-situ hybridization (CISH) on core-needle biopsies with HER2 status determined by fluorescence in-situ hybridization (FISH) on surgical specimens. The concordance between biopsy and operative results was also assessed for each method. We studied 260 breast tumors from 24 centers between April 2003 and August 2009. Excellent concordance ( $\kappa:$ 0.92-0.97) was shown between immunohistochemistry and FISH with low discordance rates (2-4\%), high specificity (97-98\%) and sensitivity values (95-99\%), with no significant difference according to the immunohistochemistry interpretation guidelines used. The correlation between SISH and $\mathrm{CISH}$ on biopsies and FISH on surgical samples was strong ( $\kappa: 0.96$ and 0.94 , respectively), with no significant difference between false negative rates or sensitivity and specificity values (2 and 5\%, 99 and $96 \%, 98$ and $98 \%$, respectively). Whatever the evaluation technique, excellent concordance between biopsies and surgical specimens was observed ( $\kappa \geqslant 0.97$; discordance rates between 1 and $2 \%$ ), with high sensitivity (98-99\%) and specificity (98-100\%). Based on these results, when FISH cannot be used, SISH and/or CISH could be proposed as an alternative method to determine HER2 status and to confirm any ambiguous immunohistochemistry results, either for preoperative percutaneous biopsies or for surgical specimens. They could also be used for quality controls and immunohistochemistry calibration. Modern Pathology (2012) 25, 675-682; doi:10.1038/modpathol.2011.201; published online 6 January 2012

Keywords: adjuvant; breast cancer; HER2; immunohistochemistry; in-situ hybridization

In non-metastatic breast cancer, knowledge of HER2 status at diagnosis is recommended to determine

Correspondence: Dr L Arnould, Département de Biologie et de Pathologie des Tumeurs, Centre Georges-François Leclerc, 1 rue Professeur Marion, BP 77980, 21079 Dijon cedex, France.

E-mail: larnould@dijon.fnclcc.fr

Received 7 July 2011; revised 24 October 2011; accepted 24 October 2011; published online 6 January 2012 the adjuvant therapy strategy. It is therefore necessary to have standardized and validated procedures for this evaluation before making any therapeutic decision.

For the HER2 status determination, core-needle biopsies may be less reliable than surgical specimens due to the smaller volume of the tissue sample, the possible sampling error on a tumor with a heterogeneous distribution of the antigens 
within the tumor, and potential crush/edge artifacts in the core-needle biopsies. However, counter arguments suggest that core-needle biopsies may be better fixed than lumpectomies and should be used to assess the patient's biomarker status. Furthermore, HER2 status is usually homogenous $(<3 \%$ of heterogeneous tumors $)^{1}$ and there is a concrete clinical need for testing core-needle biopsies insomuch as neoadjuvant therapy strategies (and then HER2 status) may be determined for both operable and non-operable breast cancers.

Regarding the available guidelines, ASCO/CAP (American Society of Clinical Oncology/College of American Pathologists) recommends that cores entirely involved by retraction artifacts or crush artifacts should not be used as a sample to perform/ interpret HER2 immunohistochemistry ${ }^{2,3}$ and UK recommendations which precise that observers should be aware of the range of common artifacts, including edge artifacts, which can be problematic in small biopsy samples. ${ }^{4}$ French guidelines authorize testing on core biopsies ${ }^{5}$ and Canadian recommendations do not specifically discuss core biopsy processing/testing. ${ }^{6}$

Recently, bright-field in-situ hybridization techniques such as chromogenic in-situ hybridization (CISH) and silver-enhanced in situ hybridization (SISH), which combine features of immunohistochemical analysis and in-situ hybridization (ISH), have been introduced for the determination of HER2 status. These new techniques allow results to be visualized by standard bright-field microscopy, and signals do not decay over time.

In most studies, a high correlation ( $>90 \%$ ) was shown between the first available procedures designed to evaluate HER2 status (immunohistochemistry, fluorescence in-situ hybridization (FISH), considered as the gold standard, and CISH, first described in 2000) ${ }^{7}$ but concordance between these methods might depend, at least in part, on centers (methodology, instrumentation and experience of the laboratories performing the testing), particularly for immunohistochemistry and FISH. ${ }^{8-11}$

SISH was implemented more recently and three studies reported a 97, 96 and $87 \%$ concordance between SISH and FISH results on excision samples $^{12-14}$ taking into account that discordant cases could be partly explained by intratumor heterogeneity of amplification. One study showed an $89 \%$ concordance of SISH results between surgical specimens and core-needle biopsies but the number of studied cases was limited $(n=56) .{ }^{15}$

Since FISH on surgical specimens is considered as the gold standard for her2 gene status evaluation in breast cancer in the ASCO/CAP guidelines, ${ }^{2,3}$ concordance with other available HER2 tests (immunohistochemistry, SISH and CISH), on coreneedle biopsies, is essential because the results could be used for patient's management, and will be the only material available in the neoadjuvant setting.

\section{Materials and methods}

\section{Study Design}

This French multicenter, cross-sectional, histopathological study was based on the diagnostic core-needle biopsies and surgical specimens, in women suffering from a previously untreated early-stage breast cancer.

Twenty histopathological laboratories (10 cancer centers, 5 university hospitals and 5 private laboratories) involved in breast cancer diagnosis participated in the study. They used immunohistochemistry and were trained to perform CISH assay. There were also four reference centers (2 cancer centers, 1 university hospital and 1 private laboratory) experienced in FISH and CISH and in the analysis of discordant cases. Among these 24 centers, 18 performed SISH assays. Participating centers had to recruit patients suffering, at first diagnosis, from non-metastatic, invasive breast carcinoma that had not been treated before surgical removal, and whose core-needle biopsies and surgical specimens were available at the center. Samples had to contain sufficient invasive tumor material to perform the different study assays, and histological tissues were fixed, depending of the structure's procedures, by neutral buffered formalin, alcohol-formalin-acetic acid, Hollande's fixative or alcohol-formalin.

It was decided to make an a priori selection of the patients, to reach significance in the statistical analysis taking into account the estimation that only $15 \%$ of patients with early breast cancer have an HER2-positive status. Patient screening was based on immunohistochemistry results obtained on surgical specimens and each center had to recruit 5-6 patients scored $3+, 2$ patients scored $2+$ and 3 patients scored $0 / 1+$, by immunohistochemistry. The retrospective deadline for the oldest selected cases was fixed at 1 January 2003. If there were insufficient retrospective cases, prospective cases could be included. Collected data were patient and tumor characteristics, HER2 evaluation method (fixative procedures, type of autostainers, antigens retrieval methods, types of antibodies, with the interpretation guideline used for immunohistochemistry; CISH, SISH or FISH procedures for hybridization techniques) according to the type of specimen (core-needle biopsy or surgical specimens), and results.

In accordance with French legislation regarding non-interventional studies, the study protocol was approved by the 'Comité Consultatif sur le Traitement de l'Information en Matière de Recherche dans le Domaine de la Santé' (Consultative Committee on Information Processing for Research in the Field of Health) and validated by the 'Commission Nationale de l'Informatique et des Libertés' (Independent administrative authority protecting privacy and personal data), which guarantees subject confidentiality. 


\section{Study Objectives}

The primary study objective was to assess the concordance of HER2 status determined by immunohistochemistry, CISH and SISH, performed on core-needle biopsies, with FISH results obtained on surgical specimens. The scoring was performed according to different guidelines used: test performed in routine practice by the participant centers (Herceptest (pathologists' scoring); cutoff of 10\% for $3+$ category $^{16}$ ), ASCO/CAP criteria (cutoff of $30 \%$ for $3+$ category $^{2}$ ), and GEFPICS (Groupe d'Etude des Facteurs Pronostiques par Immunohistochimie dans le Cancer du Sein) criteria (cutoff of $60 \%$ of positive cells intermediate intensity for $2+$ category $^{8}$ ). The secondary objectives of the study were to assess the concordance of HER2 status assessed on core-needle biopsies with that determined on surgical specimens (by immunohistochemistry, SISH, CISH and FISH), and to describe the clinical and histopathological characteristics of patients and tumors.

\section{Statistical Methods}

In all, 220 enrolled patients were needed to obtain 196 evaluable cases with the hypothesis of $15 \%$ discordant cases between CISH results on coreneedle biopsies and FISH results on surgical specimens, using a two-sided 95\% confidence interval with $5 \%$ precision.

The percentages of discordant cases between immunohistochemistry, CISH and SISH on preoperative specimens, and FISH on surgical specimens were described with their 95\% confidence intervals as well as sensitivity, specificity, false positive and negative rates, and positive and negative predictive values. Concordance results for immunohistochemistry were compared between different interpretation guidelines, using coefficient of correlation $\kappa$, as well as the correlation results with CISH and SISH. The same statistical methods were used for concordance between results of each method according to the type of tested sample (core-needle biopsies vs surgical specimens). Clinical and pathological characteristics of patients were described in the overall studied population.

All tests were two-sided with an $\alpha$ risk at $5 \%$. Statistical analyses were performed using SAS Version 8.2 (SAS Institute, Cary, NC, USA).

\section{Results}

\section{Analysis Population}

Breast tumor specimens were tested between 14 April 2003 and 27 August 2007. The analysis population is described in Figure 1. In all, 261 patients were enrolled by 24 centers including 4 referent laboratories and 20 local centers; all the 20 local centers (83\%) recruited the 11 expected patients. Among the 260 analyzed patients (1 exclusion for previous neoadjuvant treatment), 193 had also an SISH evaluation of their tumor, performed by 18 centers equipped with a Benchmark immunostainer (Ventana, Tucson, USA) according to the manufacturer guidelines.

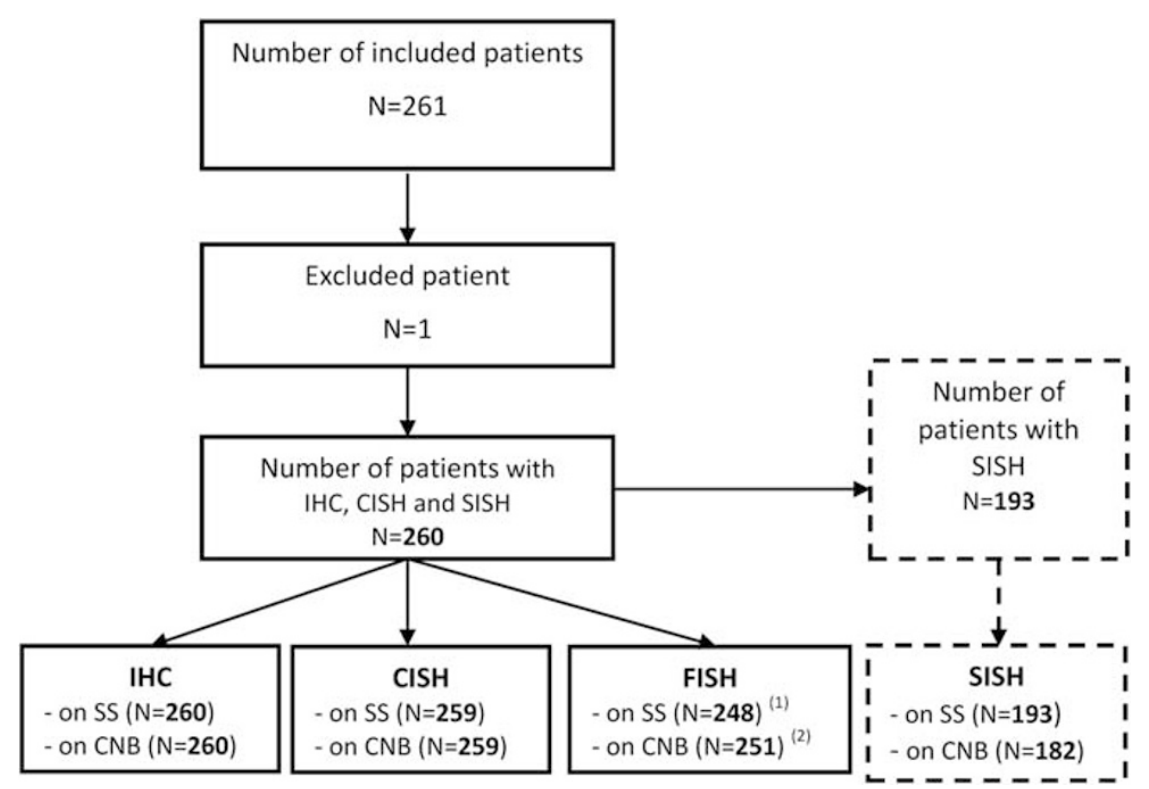

Figure 1 Study populations and data sets analyzed according to performed tests. SS, surgical specimen; CNB, core-needle biopsy; IHC, immunohistochemistry; FISH, fluorescence in-situ hybridization; CISH, chromogenic in-situ hybridization; SISH, silver in-situ hybridization. ${ }^{(1)}$ As judged by referent center, surgical specimen was not sufficient for FISH in 12 cases. ${ }^{(2)}$ As judged by referent center, preoperative biopsy specimen was not sufficient for FISH in 9 cases. 


\section{Patient and Tumor Characteristics}

Women were aged $58 \pm 13$ years at inclusion and the median time between the first diagnosis (based on the results of immunohistochemistry performed on preoperative biopsy specimen) and inclusion was 32 days.

On the basis of pretreatment and post-surgical histopathological analyses, main tumor characteristics were as follows: invasive ductal carcinoma (90\% of cases), median tumor size (18 mm (range: 3-80)), (p)TNM stage (T1 (60\%) or T2 (35\%), pNo $(61 \%))$, SBR (Scarff Bloom and Richardson) grade (I 13\%, II 43\%, III 45\%), hormonal status (ER+ (positive estrogen receptor) or $\mathrm{PR}+$ (positive progesterone receptor) $73 \%, \mathrm{ER}+$ and $\mathrm{PR}+49 \%$ ).

\section{Technical Evaluation Method of HER2 Status}

\section{Fixation of Specimens}

Whatever the test performed (immunohistochemistry, CISH, FISH or SISH), analyzed core-needle biopsies specimens were mostly fixed with a formalin-based fixative (neutral buffered formalin in 47-54\% or Hollande's fixative in 4 and $6 \%$, depending on the test), and less frequently with alcohol-based fixative (alcohol-formalin-acetic acid in 34-44\% or alcoholformalin in 4-6\%, depending on the test).

In contrast, surgical specimens were mostly fixed with alcohol-based fixative (alcohol-formalin-acetic acid in $52-54 \%$ or alcohol-formalin in 4-6\%, depending of the test) and less frequently with formalin-based fixative (neutral buffered formalin in 35-40\% or Hollande's fixative in 4-6\%, depending on the test).

Evaluation of HER2 Status with Immunohistochemistry and/or FISH

Whatever the type of sample (core-needle biopsies or surgical specimens), the applied method was fully automated in $96 \%$ of cases and partially (not the deparaffinization, nor the antigen retrieval) in $69 \%$. Slides were pretreated by using polyclonal A0485 anti-HER2 primary antibody (Dako, Glostrup, Denmark) in $\sim 75 \%$ of samples; $4 \mathrm{~B} 5$ (Ventana) and monoclonal CB11 (Novocastra, Newcastel, UK) antibodies were used less frequently $(<20$ and $<10 \%$, respectively).

Positive $(3+)$ HER2 status was observed in $50-51 \%$ of cases and negative $(0 / 1+)$ results were observed in 29-43\% of cases, depending on the immunohistochemistry interpretation guidelines used and the type of specimen (Figure 2). The rate of equivocal cases $(2+)$ ranged between 7 and $20 \%$ depending on the guideline used but it was lower for results observed on core-needle biopsies and surgical specimens when evaluated according to GEFPICS guidelines (7 and 8\%, respectively). Most often, FISH could be used to determine any immunohistochemistry equivocal cases $(2+)$, with $<3 \%$ remaining doubtful (Figure 3 ).

\section{Technical Failures}

Technical failure occurred in 6 and $5 \%$ of FISH tests, 5 and $3 \%$ of CISH tests, and 8 and $5 \%$ of SISH tests performed respectively on core-needle biopsies and surgical specimens.

\section{Concordance of HER2 Status Determined by Immunohistochemistry, CISH and SISH (on Core- Needle Biopsies) with FISH Results (on Surgical Specimens)}

Whatever the immunohistochemistry interpretation guideline applied, excellent concordance ( $\kappa$ : 0.920.97) was shown between immunohistochemistry on core-needle biopsies and FISH on surgical specimens (discordance rates: $2-4 \%$ ) (Table 1). Specificity (97-98\%) and sensitivity values (95-99\%) of

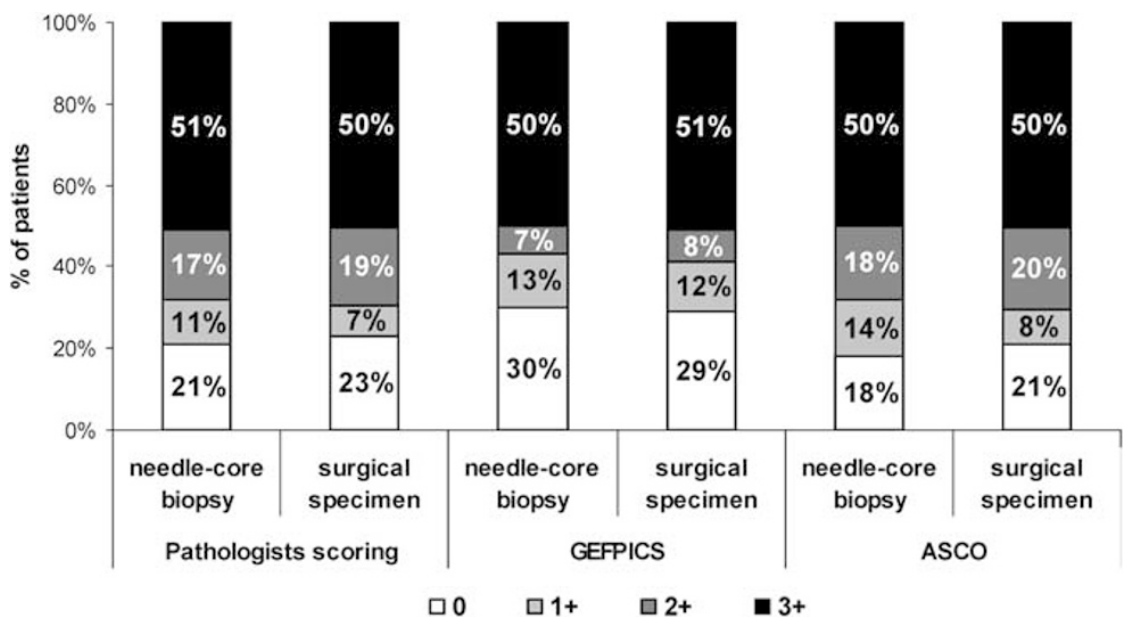

Figure 2 HER2 status according to immunohistochemistry results on preoperative biopsies and surgical specimens ( $n=260$ ). ASCO, American Society of Clinical Oncology; GEFPICS, Groupe d'Etude des Facteurs Pronostiques par Immunohistochimie dans le Cancer du Sein. 


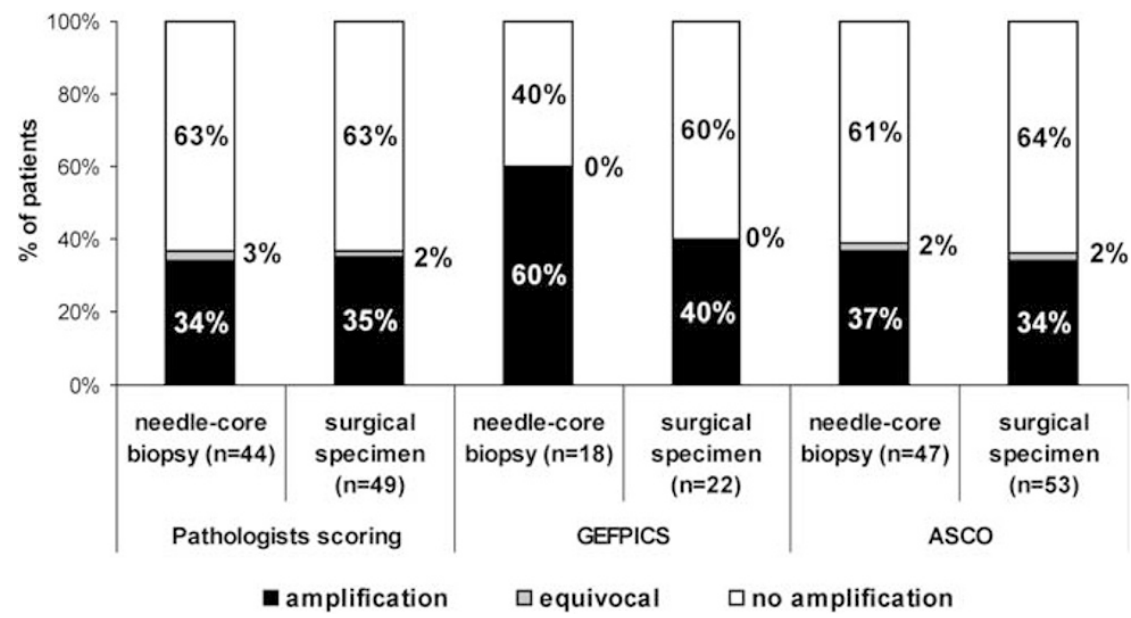

Figure 3 HER2 status of equivocal immunohistochemistry results $(2+)$ according to FISH on surgical specimens. ASCO, American Society of Clinical Oncology; GEFPICS, Groupe d'Etude des Facteurs Pronostiques par Immunohistochimie dans le Cancer du Sein.

Table 1 Concordance of HER2 status determined by IHC, CISH and SISH (on core-needle biopsy specimens) with FISH results (on surgical specimens)

\begin{tabular}{|c|c|c|c|c|c|}
\hline $\begin{array}{l}\text { HER2 status evaluation } \\
\text { method }\end{array}$ & $\begin{array}{l}\text { Discordant } \\
\text { rate } \\
\%\left[\begin{array}{lll}95 \% & C I\end{array}\right]\end{array}$ & $\begin{array}{l}\text { Sensitivity }(\mathrm{Se}) \\
\text { Specificity }(\mathrm{Sp}) \\
\quad \%[95 \% \mathrm{CI}]\end{array}$ & $\begin{array}{c}\text { Pos. (PPV) and Neg. } \\
\text { (NPV) predictive values } \\
\%[95 \% \text { CI] }\end{array}$ & $\begin{array}{c}\text { False Pos. (FPR) false } \\
\text { Neg. (FNR) rates } \\
\% \text { [95\% CI] }\end{array}$ & $\begin{array}{c}\text { Kappa } \\
\text { coefficient } \\
\%\end{array}$ \\
\hline IHC (Pathologists' scoring) & $2[0 ; 3]$ & Se $99[98 ; 100]$ & PPV $98[96 ; 100]$ & FPR $2[0 ; 4]$ & 0.97 \\
\hline 201 Conclusive cases & & Sp 97 [93; 100] & NPV 99 [96; 100] & FNR $1[0 ; 4]$ & \\
\hline IHC (ASCO) & $2[0 ; 4]$ & Se $98[96 ; 100]$ & PPV $98[96 ; 100]$ & FPR $2[0 ; 4]$ & 0.96 \\
\hline 194 Conclusive cases & & Sp $97[93 ; 100]$ & NPV 97 [93; 100] & FNR $3[0 ; 7]$ & \\
\hline IHC (GEFPICS) & $4[2 ; 7]$ & Se $95[91 ; 100]$ & PPV $98[96 ; 100]$ & FPR $2[0 ; 4]$ & 0.92 \\
\hline 230 Conclusive cases & & Sp $98[95 ; 100]$ & NPV $93[87 ; 98]$ & FNR $7[2 ; 13]$ & \\
\hline CISH & $2[0 ; 4]$ & Se $99[96 ; 100]$ & PPV $99[96 ; 100]$ & FPR $2[0 ; 4]$ & 0.96 \\
\hline 234 Conclusive cases & & Sp $98[95 ; 100]$ & NPV $98[95 ; 100]$ & FNR $2[0 ; 5]$ & \\
\hline SISH & $3[0 ; 6]$ & Se $96[92 ; 100]$ & PPV $99[96 ; 100]$ & FPR $1[0 ; 4]$ & 0.94 \\
\hline 150 Conclusive cases & & Sp $98[95 ; 100]$ & NPV $95[90 ; 100]$ & FNR $5[0 ; 10]$ & \\
\hline
\end{tabular}

IHC, immunohistochemistry; FISH, fluorescence in-situ hybridization; CISH, chromogenic in-situ hybridization; SISH, silver in-situ hybridization; ASCO, American Society of Clinical Oncology; GEFPICS, Groupe d'Etude des Facteurs Pronostiques IHC dans le Cancer du Sein.

immunohistochemistry/FISH correlations did not differ significantly according to the immunohistochemistry interpretation guidelines used. Sensitivity was particularly high when results were determined by pathologists' scoring and using ASCO guidelines (sensitivity: 99 and 98\%, respectively). Although lower, the sensitivity of immunohistochemistry results interpreted using GEFPICS guidelines (sensitivity: 95\%) were mainly related to higher false negative results $(7 \%)$ but did not differ significantly from immunohistochemistry results for sensitivity using the other guidelines.

Regarding ISH methods on core-needle biopsies, CISH and SISH showed a strong correlation with FISH on surgical specimens ( $\kappa$ : 0.96 and 0.94 , respectively), even if a lower false negative rate was observed for CISH than for SISH results (2 vs $5 \%$ ). The characteristics of CISH/FISH and SISH/ FISH correlations did not differ significantly, both methods being highly sensitive and specific in determining HER2 status on core-needle biopsies (sensitivity: 99 and 96\%—-specificity: both 98\%).

\section{Concordance of HER2 Status Determined by Each Test (Immunohistochemistry, FISH, CISH and SISH) on Core-Needle Biopsies and Surgical Specimens}

Whatever the test used, excellent concordance was shown between core-needle biopsies and surgical specimens $(\kappa \geqslant 0.97$ and discordance rates between 1 and $2 \%$ ) (Table 2).

For immunohistochemistry, although the discordance rate between core-needle biopsies and surgical specimens was higher for results interpreted using GEFPICS guidelines (2\%), it did not differ significantly from discordance rates interpreted using pathologists' scoring and ASCO guidelines (both: 1\%). Sensitivity results were similar between immunohistochemistry interpretation guidelines 
Table 2 Concordance of HER2 status determined by each test (IHC, FISH, CISH and SISH) on core-needle biopsy specimens and surgical specimens

\begin{tabular}{|c|c|c|c|c|c|}
\hline $\begin{array}{l}\text { HER2 status evaluation } \\
\text { method }\end{array}$ & $\begin{array}{c}\text { Discordant } \\
\text { rate } \\
\%\left[\begin{array}{lll}95 \% & C I\end{array}\right]\end{array}$ & $\begin{array}{l}\text { Sensitivity (Se) } \\
\text { Specificity (Sp) } \\
\%[95 \% \mathrm{CI}]\end{array}$ & $\begin{array}{l}\text { Pos. (PPV) and Neg. } \\
\text { (NPV) Predictive Values } \\
\%[95 \% \text { CI] }\end{array}$ & $\begin{array}{c}\text { False Pos. (FPR) False } \\
\text { Neg. (FNR) Rates } \\
\%\left[\begin{array}{ll}95 \% & \text { CI] }\end{array}\right.\end{array}$ & $\begin{array}{c}\text { Kappa } \\
\text { coefficient } \\
\%\end{array}$ \\
\hline IHC (Pathologists' scoring) & $1[0 ; 2]$ & Se $99[98 ; 100]$ & PPV $100[100 ; 100]$ & FPR $0[0 ; 0]$ & 0.99 \\
\hline 201 Conclusive cases & & Sp $100[100 ; 100]$ & NPV $99[96 ; 100]$ & FNR $1[0 ; 4]$ & \\
\hline IHC (ASCO) & $1[0 ; 2]$ & Se $99[98 ; 100]$ & PPV $100[100 ; 100]$ & FPR $0[0 ; 0]$ & 0.99 \\
\hline 194 Conclusive cases & & Sp $100[100 ; 100]$ & NPV $99[96 ; 100]$ & FNR $1[0 ; 4]$ & \\
\hline IHC (GEFPICS) & $2[0 ; 3]$ & Se $98[96 ; 100]$ & PPV $98[96 ; 100]$ & FPR $2[0 ; 4]$ & 0.97 \\
\hline 230 Conclusive cases & & Sp $98[95 ; 100]$ & NPV $98[95 ; 100]$ & FNR $2[0 ; 5]$ & \\
\hline FISH & $1[0 ; 1]$ & Se $99[98 ; 100]$ & PPV $100[100 ; 100]$ & FPR $0[0 ; 0]$ & 0.99 \\
\hline 220 Conclusive cases & & Sp $100[100 ; 100]$ & NPV $99[97 ; 100]$ & FNR $1[0 ; 3]$ & \\
\hline CISH & $1[0 ; 2]$ & Se $99[97 ; 100]$ & PPV $100[100 ; 100]$ & FPR $0[0 ; 0]$ & 0.98 \\
\hline 234 Conclusive cases & & Sp $100[100 ; 100]$ & NPV $98[95 ; 100]$ & FNR $2[0 ; 5]$ & \\
\hline SISH & $1[0 ; 2]$ & Se $99[96 ; 100]$ & PPV $100[100 ; 100]$ & FPR $0[0 ; 0]$ & 0.99 \\
\hline 150 Conclusive cases & & Sp $100[100 ; 100]$ & NPV $99[96 ; 100]$ & FNR $2[0 ; 4]$ & \\
\hline
\end{tabular}

IHC, immunohistochemistry; FISH, fluorescence in-situ hybridization; CISH, chromogenic in-situ hybridization; SISH, silver in-situ hybridization; ASCO, American Society of Clinical Oncology; GEFPICS, Groupe d'Etude des Facteurs Pronostiques IHC dans le Cancer du Sein.

(98\% for GEFPICS and 99\% for pathologists' scoring and ASCO) and there was no significant difference in specificity results (specificity: 100\% for pathologists' scoring and ASCO, 98\% for GEFPICS).

For FISH, CISH and SISH, discordance rates were $<1 \%$ and did not differ significantly. Correlations between core-needle biopsies and surgical specimens also showed excellent sensitivity values (99\% for all three methods, always related to false negative rates: 1,2 and $2 \%$, respectively) and $100 \%$ specificity with no significant difference observed between these correlations.

\section{Discussion}

Several studies compared HER2 status by immunohistochemistry and FISH on breast core-needle and excisional biopsies ${ }^{17-27}$ and showed generally a very good concordance between these samplings (average $\sim 90 \%$ ), suggesting that core-needle biopsies can be used with confidence for HER2 status determination. However, most of these studies assessed only a small number of samples with a limited statistical power to detect discordances, leading to considerable differences between their results (range of concordance rate: 64-100\%). More recent studies $^{28-30}$ assessing higher samples $(n=500,332$ and 225 paired samples of core-needle biopsies and subsequent surgical specimens, respectively) showed excellent concordance rates (90, 99 and $89 \%$, respectively). This is confirmed in our hands. Thus, when comparing the results of each test on core-needle biopsies to these obtained on surgical specimens, no significant difference was observed between discordance rates, sensitivity, specificity, false positive and false negative rates for immunohistochemistry, irrespective of the guidelines used, or for ISH methods (FISH, CISH and SISH).
Whatever the test performed, concordance between core-needle biopsies and surgical specimens on the same tissue sample remained excellent, $\kappa$ coefficients of correlation ranging from 0.97 (for immunohistochemistry interpreted using GEFPICS guidelines) to 0.99 (for immunohistochemistry interpreted using pathologists' scoring and ASCO guidelines or FISH, and SISH). These results, in accordance with previous findings, ${ }^{19,20,24,31,32}$ suggest that intratumoral heterogeneity of HER2 is not a significant confounding factor when analyzing small sized samples, if ISH is used. In spite of excellent concordance between core-needle biopsies and surgical specimens, some pathologists retest for HER2 status on surgical specimens in case of rare heterogeneous cases. ${ }^{32}$

Furthermore, we have shown that the nonfluorescent immunohistochemistry techniques on core-needle biopsies can generally be used with confidence, an excellent concordance rate between immunohistochemistry on core-needle biopsies and FISH on surgical specimens ( $\kappa$ : 0.92-0.97) being observed whatever the immunohistochemistry interpretation guideline used. Likewise, CISH and SISH on core-needle biopsies showed a strong correlation with FISH on surgical specimens ( $\kappa: 0.96$ and 0.94 , respectively). These results are consistent with previous data available on surgical specimens. ${ }^{12-14,33}$

In conclusion and based on our results, immunohistochemistry status assessed on cores biopsy is highly concordant with FISH in cores and surgical specimens. Furthermore, CISH and/or SISH even represents a safe alternative method to determine HER2 status on these cores biopsies to confirm any ambiguous immunohistochemistry results $(2+)$ or to perform HER2 status screening, if ISH is used as first-line screening method. CISH or SISH may also be used for calibration or quality controls of immunohistochemistry, either on cores biopsies or on surgical specimens. 


\section{Acknowledgements}

This study was sponsored by Roche. Many thanks to all pathologists for their participation in the study: Algros Marie-Paule, Hôpital Jean Minjoz, Besançon; Antoine Martine, CHU Tenon, Paris; Arbion Sylvie, Hôpital Bretonneau, Tours; Arnould Laurent, Centre Georges-François Leclerc, Dijon; Balaton André, Cabinet d'Anatomopathologie, Bièvres; Bibeau Fréderic, Centre Val d'Aurelle Paul Lamarque, Montpellier; Blanc-Fournier Cécile, Centre François Baclesse, Caen; Chenard Marie-Pierre, CHU Hautepierre, Strasbourg; Conan-Charler Virginie, Hôpital Morvan, Brest; Ettore Francette, Centre Antoine Lacassagne, Nice; Guinebretière Jean-Marc, Centre René Huguenin, Saint Cloud; Guiu Michel, Cabinet d'Anatomopathologie, Perpignan; Jacquemier Jocelyne, Institut Paoli-Calmettes, Marseille; Laberge Sophie, Centre Henri Becquerel, Rouen; LacroixTriki Magali, Institut Claudius Regaud, Toulouse, Leroux Agnès, Centre Alexis Vautrin, Vandoeuvre Les Nancy; MacGrogan Gaétan, Institut Bergonié, Bordeaux; Mathieu Marie-Christine, Institut Gustave Roussy, Villejuif; Maury Florence, Centre Médical République, Clermont-Ferrand; PenaultLlorca Frédérique, Centre Jean Perrin, ClermontFerrand; Roche-Combet Isabelle, IHCP, Le Bouscat; Roger Pascal, Hôpital Lapeyronie, Montpellier; Roignot Patrick, Cabinet d'Anatomopathologie, Dijon, Tas Patrick, Cabinet Richier, Rennes.

\section{Disclosure/conflict of interest}

Roche financially supported the development of this manuscript, and funded the research. The sponsor was responsible for study management and statistical analyses. The authors were responsible for the study design, data preparation, anatomopathological tests and interpretation, review and final approval of the manuscript before submission. All the authors received honoraria from Roche as members of an advisory board.

\section{References}

1 Simon R, Nocito A, Hubscher T, et al Patterns of her-2/ neu amplification and overexpression in primary and metastatic breast cancer. J Natl Cancer Inst 2001;93:1141-1146.

2 Wolff AC, Hammond EH, Schwartz JN, et al. American Society of Clinical Oncology/College of American Pathologists guideline recommendations for human epidermal growth factor receptor 2 testing in breast cancer. J Clin Oncol 2007;25:118-145.

3 Wolff AC, Hammond EH, Schwartz JN, et al. American Society of Clinical Oncology/College of American Pathologists guideline recommendations for human epidermal growth factor receptor 2 testing in breast cancer. Arch Pathol Lab Med 2007;131:18-43.

4 Ellis IO, Bartlett J, Dowsett M, et al. Best practice no 176. Updated recommendations for HER2 testing in the UK. J Clin Pathol 2004;57:233-237.
5 Penault-Llorca F, Vincent-Salomon A, Bellocq JP, et al. Update of the GEFPICS' recommendations for HER2 status determination in breast cancers in France. Ann Pathol 2010;30:357-373.

6 Hanna W, O'malley FP, Barnes P, et al. Updated recommendations from the Canadian National Consensus Meeting on HER2/neu testing in breast cancer. Curr Oncol 2007;14:149-153.

7 Tanner M, Gancberg D, Di Leo A, et al. Chromogenic in situ hybridization: a practical alternative for fluorescence in situ hybridization to detect HER-2/ neu oncogene amplification in archival breast cancer samples. Am J Pathol 2000;157:1467-1472.

8 Vincent-Salomon A, MacGrogan G, Couturier J, et al. Calibration of immunohistochemistry for assessment of HER2 in breast cancer: results of the French multicentre GEFPICS study. Histopathology 2003;42:337-347.

9 Vincent-Salomon A, MacGrogan G, Couturier J, et al. Groupe d'Etude des Facteurs Pronostiques par Immunohistochime dans les Cancers du Sein. Re: HER2 testing in the real world. J Natl Cancer Inst 2003; 95:628.

10 Roche PC, Suman VJ, Jenkins RB, et al. Concordance between local and central laboratory HER2 testing in the breast intergroup trial N9831. J Natl Cancer Inst 2002;94:855-857.

11 Paik S, Bryant J, Tan-Chiu E, et al. Real-world performance of HER2 testing: National Surgical Adjuvant Breast and Bowel Project experience. J Natl Cancer Inst 2002;94:852-854.

12 Papouchado BG, Myles J, Lloyd RV, et al. Silver in situ hybridization (SISH) for determination of HER2 gene status in breast carcinoma. Comparison with FISH and assessment of interobserver reproducibility. Am J Surg Pathol 2010;34:767-766.

13 Dietel M, Ellis IO, Höfler H, et al. Comparison of automated silver enhanced in situ hybridisation (SISH) and fluorescence ISH (FISH) for the validation of HER2 gene status in breast carcinoma according to the guidelines of the American Society of Clinical Oncology and the College of American Pathologists. Virchows Arch 2007;451:19-25.

14 Capizzi E, Gruppioni E, Grigioni AD, et al. Real time RT-PCR approach for the evaluation of ERBB2 overexpression in breast cancer archival samples: a comparative study with FISH, SISH, and Immunohistochemistry. Diagn Mol Pathol 2008;17:220-226.

15 Shousha S, Peston D, Amo-Takyi B, et al. Evaluation of automated silver-enhanced in situ hybridization (SISH) for detection of HER2 gene amplification in breast carcinoma excision and core biopsy specimens. Histopathology 2009;54:248-253.

16 Penault-Llorca F, Balaton A, Sabourin JC, et al. Immunochemistry evaluation of HER2 status in infiltration breast cancer: technical protocol and interpretation guidelines. Ann Pathol 2002;22:150-157.

17 Lowe AC, Roa N, Shintaku P, et al. Are FISH HER-2/ neu results the same on core needle biopsy and excision in patients with breast cancer [abstract]. Mod Pathol 2010;23:55A.

18 Tsuda H, Kurosumi M, Umemura S, et al. HER2 testing on core needle biopsy specimens from primary breast cancers: interobserver reproducibility and concordance with surgically resected specimens. BMC Cancer 2010;10:534.

19 D’Alfonso T, Liu YF, Monni S, et al. Accurately assessing HER-2/neu status in needle core biopsies in 
breast cancer patients in the era of neoadjuvant therapy: emerging questions and considerations addressed. Am J Surg Pathol 2010;34:575-581.

20 Apple SK, Lowe AG, Rao PN, et al. Comparison of fluorescent in situ hybridization HER-2/neu results on core needle biopsy and excisional biopsy in primary breast cancer. Mod Pathol 2009;22:1151-1159.

21 Park SY, Kim KS, Lee TG, et al. The accuracy of preoperative core biopsy in determining histologic grade, hormone receptors, and human epidermal growth factor receptor 2 status in invasive breast cancer. Am J Surg 2009;1997:266-269.

22 Cahill RA, Walsh D, Landers RJ, et al. Preoperative profiling of symptomatic breast cancer by diagnostic core biopsy. Ann Surg Oncol 2006;13:45-51.

23 Streibel JM, Bhargava R, Horbinski C, et al. The equivocally amplified HER2 FISH result on breast core biopsy. Anat Pathol 2008;129:383-390.

24 Chivukula M, Bhargava R, Brufsky A, et al. Clinical importance of HER2 immunohistologic heterogeneous expression in core-needle biopsies vs resection specimens for equivocal (immunohistochemical score 2+) cases. Mod Pathol 2008;21:363-368.

25 Usami S, Moriya T, Amari M, et al. Reliability of prognostic factors in breast carcinoma determined by core needle biopsies. Jpn J Clin Oncol 2007;27: 250-255.

26 Burge CN, Chang HR, Apple SK. Do the histologic features and results of breast cancer biomarker studies differ between core biopsy and surgical excision specimens? Breast 2006;15:167-172.
27 Erggelet J, Robe R, Holzhausen HJ, et al. Correlation of human epidermal growth factor receptor 2 (HER2), estrogen receptor (ER), and progesterone receptor (PR) expression as predicted by core biopsy with the immunohistochemical results of surgical breast cancer specimens. Breast Care 2007;2:94-98.

28 Lebeau A, Turzynski A, Braun S, et al. Reliability of human epidermal growth factor receptor 2 immunohistochemistry in breast core needle biopsies. J Clin Oncol 2010;28:3264-3270.

29 Arnedos M, Nerurkar A, Osin P, et al. Discordance between core needle biopsy (CNB) and excisional biopsy (EB) for estrogen receptor (ER), progesterone receptor (PgR) and HER2 status in early breast cancer (EBC). Ann Oncol 2009;20:1948-1952.

30 Tamaki K, Sasano H, Ishida T, et al. Comparison of core needle biopsy (CNB) and surgical specimens for accurate preoperative evaluation of ER, PgR and HER2 status of breast cancer patients. Cancer Sci 2010;101:2074-2079.

31 Taucher S, Rudas M, Mader RM, et al. Prognostic markers in breast cancer: the reliability of HER2/neu status in core needle biopsy of 325 patients with primary breast cancer. Wien Klin Wochenschr 2004;116:26-31.

32 Bilous M. Breast core needle biopsy: issues and controversies. Mod Pathol 2010;23:536-545.

33 Wolf V, Große R, Erggelet J, et al. The reliability of HER2-status determination from core-needle-biopsies and surgical specimens: a comparison of two established test methods (IHC, CISH) [abstract]. J Clin Oncol 2009;27:15S. 